\title{
Ambulatory motility patterns of the transposed short segment colon
}

\author{
G Peppas, H R Payne, K Jeyasingham
}

\begin{abstract}
Ambulatory manometry and pH-metry were performed on 10 asymptomatic patients who had undergone lower oesophageal replacement with the left hemicolon between two and 20 years previously. Recording of the ambient pH in the intrathoracic colon was carried out simultaneously. In every patient the recording, which lasted approximately eight hours, included upright, supine, prandial, and postprandial periods. The results were analysed both visually and by computer. The emerging motility patterns showed three basic types of wave, based on amplitudes - low amplitude contractions (LAC) measuring $<60 \mathrm{~mm} \mathrm{Hg}$, medium amplitude contractions (MAC) of 60$100 \mathrm{~mm} \mathrm{Hg}$, and high amplitude contractions (HAC) of 100-200 mm Hg. Additional subtypes were identified in the LACs and MACs. Using software primarily designed for oesophageal motility, the proportion of propulsive $(6 \%$ $55 \%$ ) waves could be distinguished from simultaneous or mixed waves, or both. A proportion of propulsive waves was noted in patients who had undergone surgery 10 or more years previously. It is concluded that the transposed colon retains its normal motility pattern but some adaptation to its new location may occur in the long term.

(Gut 1993; 34: 1572-1575)
\end{abstract}

Replacement of the undilatably strictured lower oesophagus by a short segment of left hemicolon

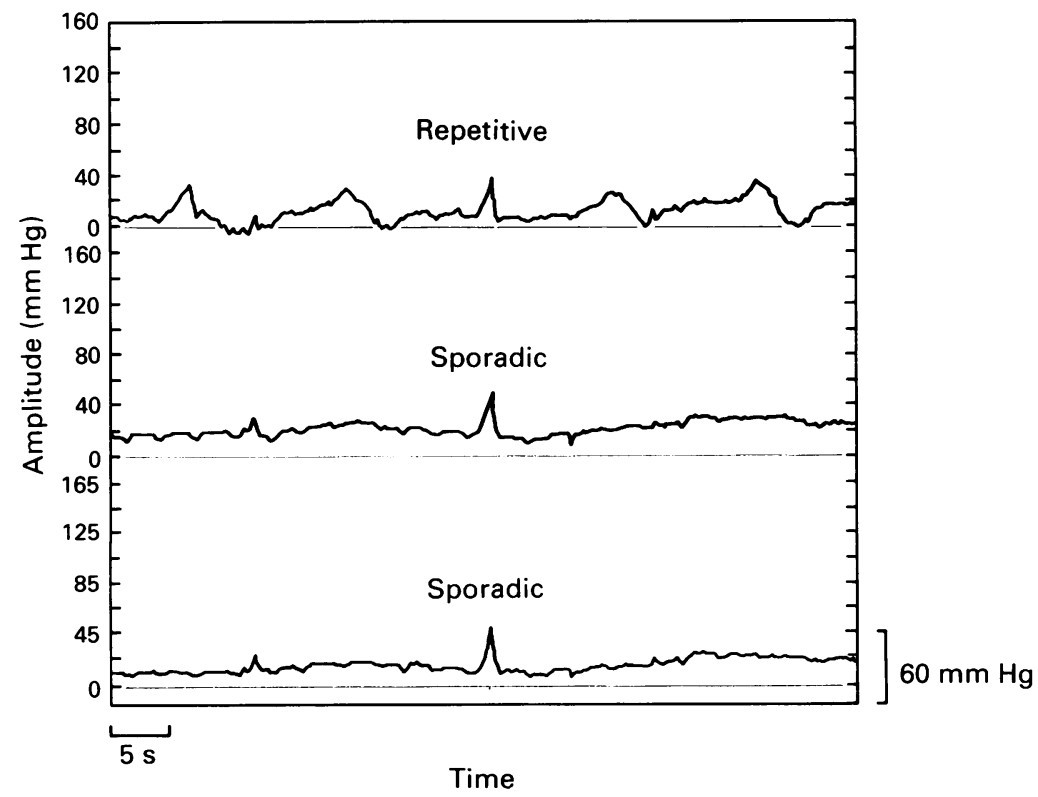

Figure 1: Low amplitude contractions of repetitive and sporadic types occurring at different levels in the transposed colonic segment. There does not seem to be a propagation of the waves along the length of the bowel. A common cavity effect may account for one of the waves occurring simultaneously at all three levels. has been practised for several decades. ${ }^{1-4}$ The motility of the left hemicolon in its normal position has been studied only in recent years. Some patterns of normal colonic motility have begun to emerge, but specific abnormalities are yet to be defined. ${ }^{5}$ The motility patterns of transposed colonic segments have until now been studied by static manometry. ${ }^{6}$ There is no information on the ambulatory motility patterns of transposed segments of colon in their new location between the oesophagus and stomach, functioning as a neo-oesophagus.

\section{Patients and methods}

Ten asymptomatic patients who had had short segment colonic interposition of the oesophagus two to 20 years previously for undilatable peptic stricture, underwent ambulatory manometric recordings in their short colonic segments. Their mean age at operation was 48 years, with a range of 21 to 67 . The operative technique entailed resection of a benign peptic oesophageal stricture and the transposition of an 18 to $24 \mathrm{~cm}$ segment of left hemicolon pedicled on the upper left colic arterial branch, between the oesophagus and stomach, with a proximal subaortic oesophagocolic anastomosis and a distal subdiaphragmatic cologastric anastomosis. A minimum length of 12 to $18 \mathrm{~cm}$ of colon was maintained below the diaphragm. Ambulatory manometry lasting approximately eight hours was performed with a Gaeltec (Dunvegan, Isle of Skye IV55 8GU) manometric catheter with three miniaturised solid state transducers placed $5 \mathrm{~cm}$ apart in its distal $10 \mathrm{~cm}$, connected proximally to a Synectics (215 Willow Road, Enfield, Middlesex) multichannel microdigitrapper. The terminal tip of the probe was positioned immediately above the diaphragm at approximately $38 \mathrm{~cm}$ from the nostril. In this way it was possible to ensure that all three transducers were located in the supradiaphragmatic part of the transposed colon. The data were analysed both visually and with the help of the Synectics Gastrosoft Multigram software (version 5), which was originally designed for oesophageal manometry. Any waves with amplitudes greater or equal to $20 \mathrm{~mm} \mathrm{Hg}$ were included in the analysis. The amplitude, wave duration, motility index (contractions/minute $x$ mean amplitude of contraction $\times$ mean duration of contration), wave frequency, and wave velocity were analysed in the upright and supine positions and during prandial and postprandial periods. An analysis was also made of the proportion of propulsive waves.

Results

The general pattern of activity consisted of periods of quiescence alternating with periods of 


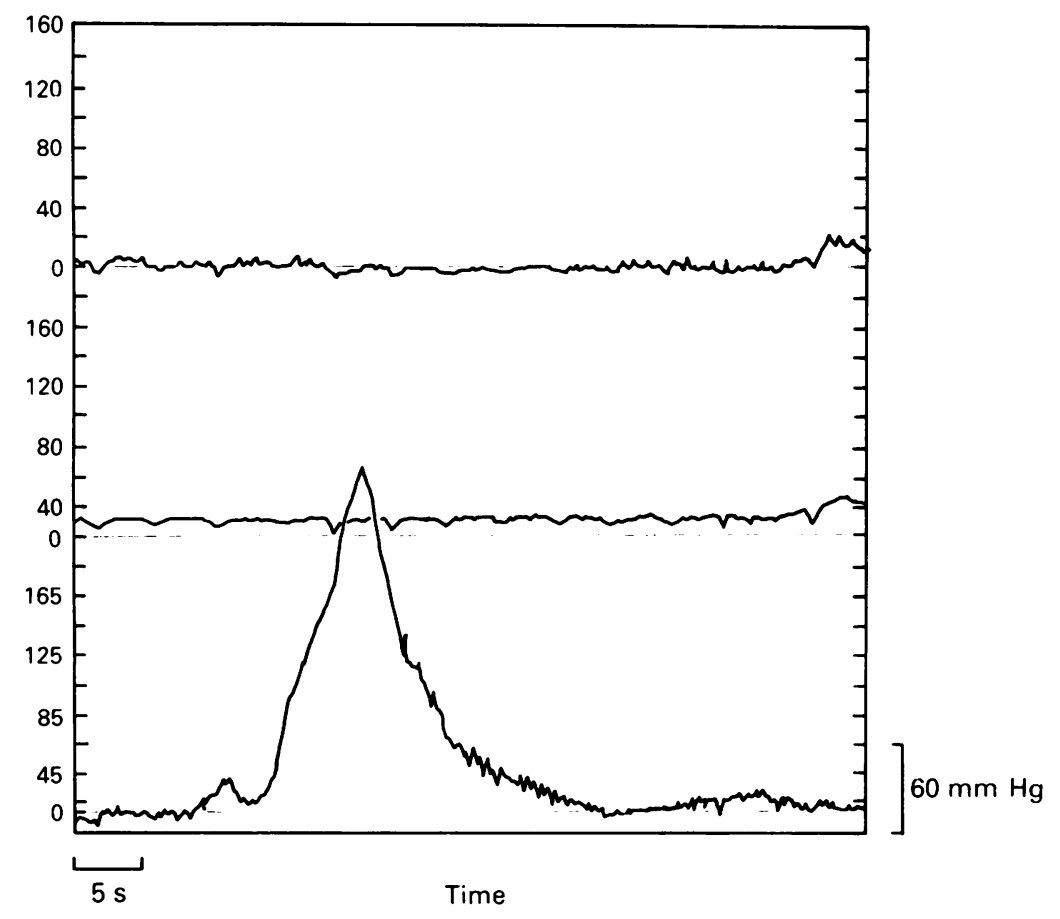

Figure 2: Non-propagated contractions measuring over $100 \mathrm{~mm} \mathrm{Hg}$ and sometimes of more than 15 seconds duration that occurred after breakfast.

activity. Based on the amplitude of contraction, we were able to recognise three types of waves as follows:

(1) Low amplitude contractions (LACs) measuring $<60 \mathrm{~mm} \mathrm{Hg}$ (Fig 1); (2) Medium amplitude contractions (MACs) measuring 60-100 mm Hg; (3) High amplitude contractions (HACs) measuring $>100 \mathrm{~mm} \mathrm{Hg}$ (Fig 2). Further subtypes were also identified as follows:

(1) The LACs showed sporadic (amplitude 40 $\mathrm{mm} \mathrm{Hg}$ and duration seven seconds), repetitive (same amplitude and duration but lasting approximately five minutes), and bursting (amplitude $25 \mathrm{~mm} \mathrm{Hg}$, duration five seconds, and bursts lasting five to 20 minutes) waves (Figs 1 and 3 ).

(2) The MACs showed two subtypes (Figs 4 and 5), a peaked variety (duration eight seconds) and a bell shaped one (duration 13 seconds).

In addition to these, HACs with durations of up to 15 seconds were seen in three patients and

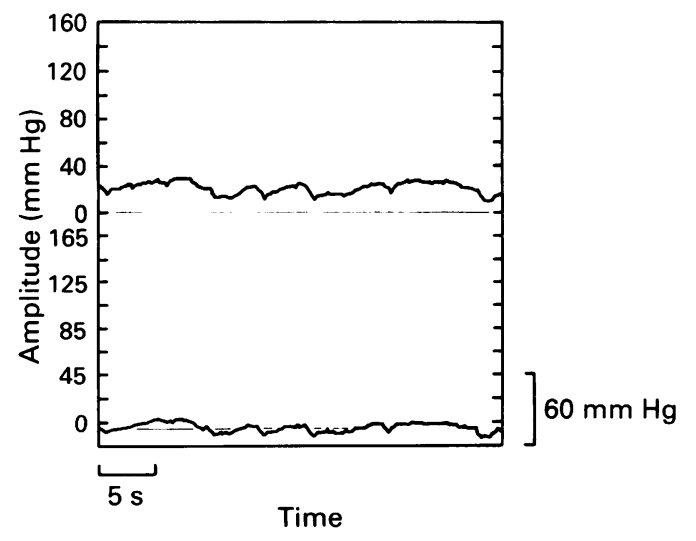

Figure 3: A tracing of the pressure recording at two levels of the transposed colon showing a bursting type of low amplitude contractions.

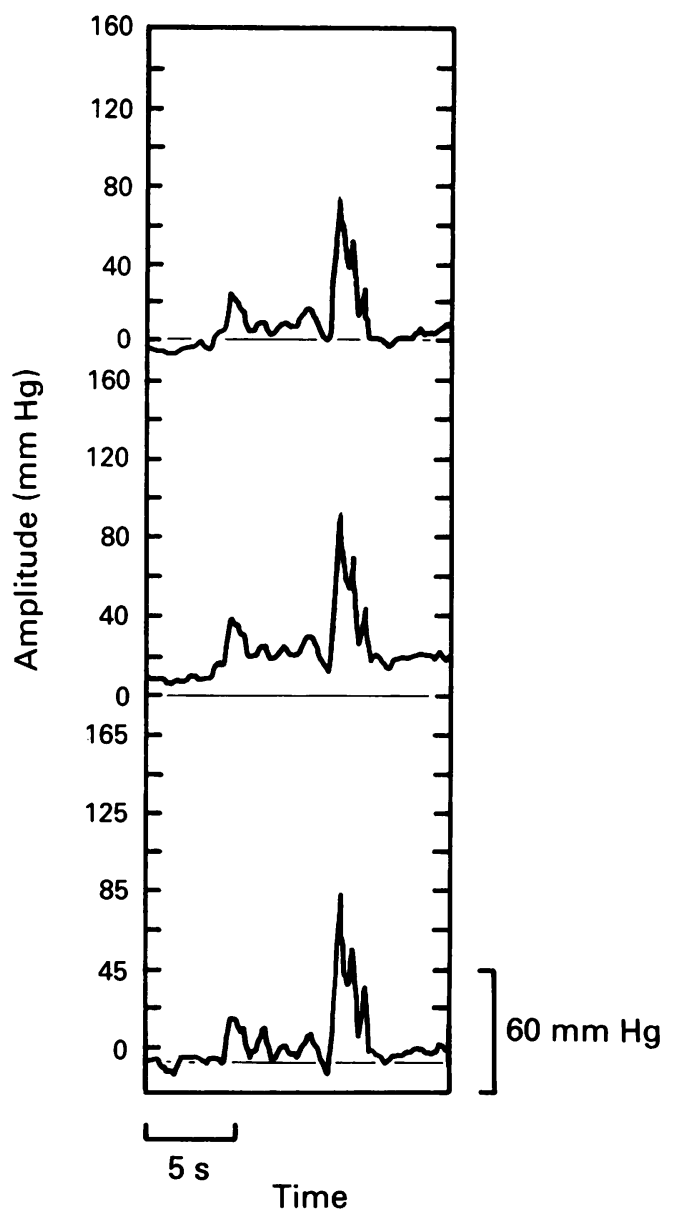

Figure 4: Simultaneous, narrow based peaked variety of medium amplitude contractions seen at all three levels.

these tended to occur mostly between 10 and $11 \mathrm{am}$.

In two patients a plateau wave with an amplitude of $20 \mathrm{~mm} \mathrm{Hg}$ that lasted $20-30$ seconds was noted postprandially (2-3 pm).

The mean wave frequency was $2.36 \mathrm{c} / \mathrm{min}$ (range $0 \cdot 1-12 \cdot 4$ ) with the higher values occurring during the prandial periods (Table I), although this did not reach statistical significance. The mean wave velocity was $1 \cdot 2 \mathrm{~cm} / \mathrm{s}$. The mean percentage of propulsive waves was $26 \cdot 6$. The average hourly motility index for all periods is shown in Table II. The difference in motility indices for the total period and the postprandial period did not reach statistical significance. None of the eight recordings incorporated a sleep period of any duration. There was, however, no correlation between the motility patterns and the short postprandial periods of sleep (after lunch).

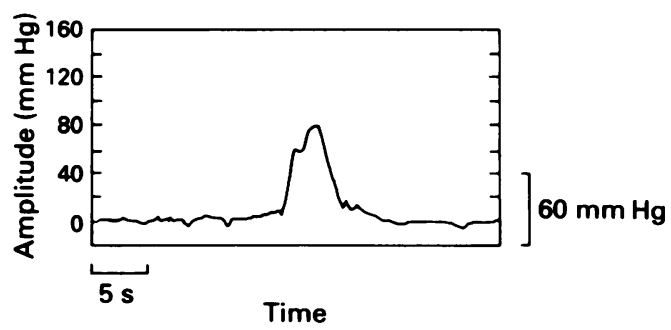

Figure 5: A tracing showing a broad based, bell shaped medium amplitude contraction. 
TABLE I Mean (SD) wave frequencies (c/min) in relation to feeding time and to posture. Statistical analysis ${ }^{\star}$ failed to show any significance between any two of the mean values

\begin{tabular}{llllll}
\hline & Total & Prandial & Postprandial & Upright & Supine \\
\hline Mean & $2 \cdot 36$ & 4.98 & 2.57 & 2.67 & $\begin{array}{l}3.42 \\
\text { (SD) }\end{array}$ \\
$(2 \cdot 00)$ & $(3.66)$ & $(2 \cdot 15)$ & $(2.06)$ & $(2 \cdot 65)$ \\
\hline
\end{tabular}

^Student's $t$ test $\mathrm{p}<0 \cdot 1$

TABLE II Mean $(S D)$ hourly motility indices in relation to feeding time and to posture $(\mathrm{mm} \mathrm{Hg} \times \operatorname{sex} \times 10(-3))$. No significance ${ }^{\star}$ could be shown between any two of the values

\begin{tabular}{llllll}
\hline & Total & Prandial & Postprandial & Upright & Supine \\
\hline Mean & $10 \cdot 82$ & $21 \cdot 14$ & $9 \cdot 58$ & 10.73 & $\begin{array}{l}10 \cdot 43 \\
(\text { SD) }\end{array}$ \\
$(7 \cdot 81)$ & $(15 \cdot 49)$ & $(7 \cdot 86)$ & $(8.04)$ & $(10.02)$ \\
\hline
\end{tabular}

$\star$ Student's $t$ test $\mathrm{p}<0 \cdot 1$

It was also not possible to correlate the motility of the exercise periods during the recording as patients were not subjected to any strenuous form of exercise during the recordings.

\section{Discussion}

The normal colonic motility patterns described by Narducci $e t a l^{5}$ consist of periods of motor quiescence irregularly alternating with periods of activity. Sleep promotes quiescence, while walking promotes motor activity.

Two types of LACs have been described, sporadic waves and those that occur in bursts. To this we have added a third variety - repetitive contraction. The LACs usually have a propulsive rate of $10 \%$ and a frequency of $3-8 / \mathrm{min}$. They occur more often in the postprandial period. In dogs, LAC type activity has been recorded originating from the interstitial cells of Cajal located at the submucosa-circular muscle interface of the colonic wall, where pacemaking takes place. ${ }^{78}$ It is also thought that a proper coupling of electrical and metabolic activity is a prerequisite for the generation of pacemaker activity. ${ }^{9}$ The extent to which sporadic, bursting, or repetitive waves occur may well be an index of the proper coupling of electrical and metabolic activity. The same factor may also account for the variation in amplitudes and frequencies noted by us and by Sunshine et $_{\text {al }}{ }^{10}$

HACs have an amplitude of $100-200 \mathrm{~mm} \mathrm{Hg}$. They are isoperistaltic but occur very infrequently, at 4.4 waves $/ 24 \mathrm{~h}$. They are seen predominantly in the early hours of the morning, or in the late postprandial period, ${ }^{511}$ and are usually followed by a second or third wave within a short period. They are promoted by colonic distension and are associated with the urge to defaecate. They may well have a reflex aetiology with an afferent arc situated in the colonic wall as well as in the gastric wall - not unlike the gastrocolic reflex. In this respect it is interesting to note that the new location of the interposed colon has not in any way altered the inherent motility characteristics of that segment.

The three main types of contractions and five subtypes that we have noted have both similarities and dissimilarities to those described by Narducci $e t a l^{5}$ in the left hemicolon in its normal location. The main difference is that we describe a MAC with an amplitude of $60-100 \mathrm{~mm} \mathrm{Hg}$ in addition to the LAC and HAC described by them. Our MAC does not conform to the amplitude criteria of the LAC and HAC respectively. We postulate that the supradiaphragmatic segment of transposed colon has evolved a MAC type activity in an attempt to overcome the 'obstructive' effect of the diaphragmatic hiatus. This factor may also account for the repetitive waves in the transposed colon.

There is controversy over the point of entry of the pelvic parasympathetic nerves into the colonic wall. ${ }^{7-9}$ Two modes of entry have been suggested, one that they pierce the muscular layer of the upper rectum and travel upwards in the wall of the colon, and the other that they follow the course of the inferior mesenteric artery and its branches. Should the first theory be correct, the similarities that exist between the motility of the colon in the normal location and in the transposed location would not exist, as the nerves would have been interrupted during transection and mobilisation of the segment of colon. Our findings support the view that the nerves follow the course of the artery and its branches which anatomically penetrate the colonic wall at various levels along the length of the bowel. During mobilisation, the marginal artery and its branches are preserved, and form the pedicle for the vascular supply of the colon in its new location.

The highest wave frequency and motility index were noted in the postprandial period. Despite the lack of statistical significance it seems that contact with food provided the most potent stimulus to inducing motor activity. The percentage propulsive rate $(26.6 \%)$ in the interposed colon is significantly lower than that seen in the normal oesophagus ( $80 \%)$, and more than double that seen in the left hemicolon in its normal location (10\%). Our findings differ from those reported by Paris et $a l^{6}$ who performed instant and not ambulatory manometry. They obtained propulsive rates of $87 \%$ and $93 \%$ when swallowing liquids and solids respectively. As Paris et al performed their measurements by instant manometry using the solid or liquid bolus, the difference between their results and ours is entirely attributable to the positive effect of the arrival of the liquid or solid bolus in the segment of colon in their studies, while in our patients the propulsive waves were in response to the contents of the colonic segment at the time. We did, however, note that patients in whom the interposition had been performed 10 or more years previously, showed a higher propulsive rate. We conclude that the transposed left hemicolon retains its normal motility patterns albeit with some minor modifications, in keeping with its new location and function as a neooesophagus. There is, however, a need for further studies in this field in order that the picture could be elucidated further.

1 Belsey HR. Reconstruction of the oesophagus with left hemicolon. F Thorac Cardiovasc Surg 1965; 49: 33-53.

2 Waterstone D. Colonic replacement of the oesophagus (intrathoracic). Surg Clin North Am 1964; 44: 1441.

3 Kelling G. Oesophagoplastik mit Hilfe des Querkolon. Zentralbl Chir 1991; 38: 1209

4 Vulliet H. De 180 oesophagoplasties et de ses diverse modifications. Semaine Med 1911; 45: 529

5 Narducci F, Bassotti G, Gaburri M, Morelli A. Twenty four 
hour manometric recording of colonic motor activity. Gut 1987; 28: 17-25

6 Paris F, Tomas-Ridocci M, Galan G, Ibor PJ, Penalver JC, Moreno E, et al. The colon as oesophageal substitute in nonmalignant disease. Eur 7 Cardio-Thorac Surg 1991; 5: 474-8. 7 Barajas-Lopez C, Berezin I, Daniel EE, Huizinga JD. Pacemaker activity recorded in interstitial cells of Cajal of Pacemaker activity recorded in interstitial cells of Cajal of the gastrointestinal

8 Sarna SK. Physiology and pathophysiology of colonic activity (Part one of two). Dig Dis Sci 1991; 36: 827-62.

9 Serio R, Barajas-Lopez C, Daniel EE, Berezin I, Hiuzinga JD.
Slow wave activity in colon: role of network of submucosal interstitial cells of Cajal. Am f Physiol 1991; 260: 6636-45. 10 Sunshine AG, Perry R, Reynolds JC, Cohen S, Ouyang A. Colonic slow wave analysis. Limitations of usefulness of the fast fourier transform (FFT). Dig Dis Sci 1989; 34: 1173-9. 11 Bassotti G, Gaburri M. Manometric investigation of high amplitude propagated contractile activity of the human amplitude propagated contractile activity of the
colon. Am f Physiol 1988; 255 (5 part 1): G660-4

12 Moreno-Osset E, Bazzocchi G, Lo S, Trombley B, Ristow E Reddy SN, et al. Association between postprandial changes in colonic intraluminal pressure and transit. Gastroenterolog 1989; 96: 1265-73. 